\title{
Backward Nonlinear Smoothing Diffusions
}

\author{
Brian D.O. Anderson*1, Adrian N. Bishop ${ }^{2}$, Pierre Del Moral ${ }^{\dagger 3}$, and Camille Palmier ${ }^{4}$ \\ ${ }^{1}$ Research School of Electrical, Energy and Material Engineering, Australian National University, Canberra, \\ Australia; and also with Hangzhou Dianzi University, China, and Data61-CSIRO in Canberra, Australia. \\ ${ }^{2}$ CSIRO \& University of Technology Sydney (UTS), Australia \\ ${ }^{3}$ INRIA, Bordeaux Research Center, Talence \& CMAP, Polytechnique Palaiseau, France \\ ${ }^{4}$ Institut de Mathématiques de Bordeaux (IMB), Bordeaux University \& ONERA Palaiseau, France
}

\begin{abstract}
We present a backward diffusion flow (i.e. a backward-in-time stochastic differential equation) whose marginal distribution at any (earlier) time is equal to the smoothing distribution when the terminal state (at a latter time) is distributed according to the filtering distribution. This is a novel interpretation of the smoothing solution in terms of a nonlinear diffusion (stochastic) flow. This solution contrasts with, and complements, the (backward) deterministic flow of probability distributions (viz. a type of Kushner smoothing equation) studied in a number of prior works. A number of corollaries of our main result are given including a derivation of the time-reversal of a stochastic differential equation, and an immediate derivation of the classical Rauch-Tung-Striebel smoothing equations in the linear setting.
\end{abstract}

Keywords: Nonlinear filtering and smoothing; Kalman-Bucy filter; Rauch-Tung-Striebel smoother; particle filtering and smoothing; diffusion equations; stochastic semigroups; backward stochastic integration; backward Itô-Ventzell formula; time-reversed stochastic differential equations; Zakai and Kushner-Stratonovich equations.

Mathematics Subject Classification: 60G35; 62M20; 93E11; 93E14; 60J60.

\section{Introduction}

Let $\left(W_{t}, V_{t}\right) \in\left(\mathbb{R}^{p} \times \mathbb{R}^{q}\right)$ be a $(p+q)$-dimensional Brownian motion for finite $p, q \geq 1$. Consider a signal-observation model $\left(X_{t}, Y_{t}\right) \in\left(\mathbb{R}^{m} \times \mathbb{R}^{n}\right)$ given by the Itô stochastic differential equation:

$$
\left\{\begin{aligned}
d X_{t} & =a_{t}\left(X_{t}\right) d t+\sigma_{t}\left(X_{t}\right) d W_{t} \\
d Y_{t} & =b_{t}\left(X_{t}\right) d t+\varsigma_{t} d V_{t}
\end{aligned}\right.
$$

for some measurable functions $\varsigma_{t}, a_{t}(x), \sigma_{t}(x), b_{t}(x)$ with appropriate dimensions. We set $Y_{0}=0$ and let $X_{0}$ be an initial random variable with absolute moments of any order. We let $\alpha_{t}(x):=\sigma_{t}(x) \sigma_{t}^{\prime}(x)$, and $\beta_{t}:=\varsigma_{t} \varsigma_{t}^{\prime}$, where $A^{\prime}$ denotes the transpose of some matrix $A$.

*B.D.O. Anderson was supported by the Australian Research Council (ARC) via grant DP160104500 and grant DP190100887; and by Data61-CSIRO.

${ }^{\dagger}$ P. Del Moral was supported in part by the Chair Stress Test, RISK Management and Financial Steering, led by the French Ecole Polytechnique and its Foundation and sponsored by BNP Paribas. 
To avoid unnecessary technical details, we assume $\beta_{t} \geq \epsilon I$, for some $\epsilon>0$, where $I$ denotes the identity matrix. We also assume the drift and sensor functions $\left(a_{u}(x), b_{u}(x)\right)$, as well as the diffusion matrix $\sigma_{u}(x)$, are smooth w.r.t. $(u, x)$ and they have uniformly bounded derivatives w.r.t $x$ of all order on $(u, x) \in[s, t] \times \mathbb{R}^{m}$, for any $s \leq t$.

These technical conditions ensure that the above stochastic differential equation (1.1) has a global solution $\left(X_{t}, Y_{t}\right)$ in the sense of Itô. In addition, $\left(X_{t}, Y_{t}\right)$ as well as the sensor function $b_{t}\left(X_{t}\right)$ have absolute moments of any order. The stochastic flow associated with the signal is also smooth w.r.t. its initial condition, and its derivatives have absolute moments of any order.

The filtering problem then consists of computing the conditional distribution $\pi_{t}$ of the random signal states $X_{t}$ of the signal given the sigma-field $\mathcal{Y}_{t}=\sigma\left(Y_{s}, s \leq t\right)$ generated by the observations. The smoothing problem is to compute the conditional distribution $\pi_{t, s}$ of the random signal states $X_{s}$ given $\mathcal{Y}_{t}$, with $t \geq s$. With this notation, we have $\pi_{t, t}=\pi_{t}$.

The filtering and smoothing problems have been studied extensively, and the literature on this topic is too broad to survey in detail here; and a review of this type is beyond the rather narrow scope of our contribution. We may point to the general texts [28, 5] for broad coverage of these problems.

We do note some rather seminal early literature in the linear setting [6, 37, 16, 39] and the nonlinear setting [6, 27, 1, 3]. The first work on the smoothing topic is the maximum likelihood solution in [6] in both the linear and nonlinear setting. The study of [37] more formally confirms the linear result in [6] and also provides a simpler formulation for the mean and covariance of the smoothing distribution. In the nonlinear setting, the work of [1, 27] introduces an analogue of a type of Kushner-Stratonovich equation (see [5] for this equation in the filtering context) for the smoothing problem. More specifically, [1, 27] propose a deterministic partial differential equation that describes the flow of the smoothing distribution in terms of a backward flow and the standard filtering distribution which acts as the boundary condition (the latter follows from the classical Kushner-Stratonovich equation).

In Section 2 we state the main contribution of this work. Our main result asserts a backward diffusion flow (i.e. a backward stochastic differential equation) whose marginal distribution at any time $0 \leq s \leq t$ is equal to the smoothing distribution $\pi_{t, s}$ when the terminal state is distributed according to the filtering distribution $\pi_{t}$.

This is a novel interpretation of the smoothing solution in terms of a nonlinear diffusion (stochastic) flow (in the spirit of McKean-Vlasov-type processes). This solution contrasts with, and complements, say, the (backward) deterministic flow of probability distributions (viz. a type of Kushner smoothing equation) in [1, 27]. We also provide a number of corollaries of our main result in Section 2.1 including an immediate derivation of the Rauch-Tung-Striebel smoothing equations [37] in the linear setting.

A number of auxiliary contributions are set forth in order to prove our main contribution to the smoothing problem. As is typical, (e.g. see [6, 37, 16, 39, 27, 1, 3, 34]), our smoothing solution requires the formulation of a related filtering problem. In Section 3 we present a brief review of the Kallianpur-Striebel formula. We then provide a novel and more direct approach to deriving weakversions of the Zakai and the Kushner-Stratonovich equations in Sections 3.1 and 3.2 respectively. We also consider the backward versions of these equations in Section 3.3.

Our approach to the filtering equations in this article combines forward and backward Itô formulas for stochastic transport semigroups with a recent backward version of the Itô-Ventzell formula presented in [13]. This semigroup methodology can be seen as an extension, to the Zakai and Kushner-Stratonovich equations, of the forward-backward stochastic analysis of diffusion flows developed in [10, 11, 13, 23, 24]. 
Our direct semigroup approach to the forward/backward filtering equations in this work contrasts with classical stochastic partial differential methods and functional analysis in Sobolev spaces; see e.g. the seminal works by Pardoux [31, 32, 34, as well as Krylov and Rozovskii [19, 20, Related reverse time diffusions and filtered and smoothed densities are also developed in [2, 3] using discrete time approximation techniques, without a detailed discussion on the existence of these densities. We present a number of auxiliary results in this direction throughout Section 3 which are utilised in the proof of our main smoothing result in Section 4 .

\subsection{Some preliminary notation}

This subsection presents some notation needed from the onset.

The signal and the observation defined in (1.1) are column vectors. Unless otherwise stated, we use the letters $f$ and $g$ to denote bounded scalar measurable test functions on some measurable space.

We denote by $\nabla f$ the column gradient whenever $f$ is a differentiable function on some Euclidian space, and by $\nabla^{2} f$ the Hessian matrix whenever it is twice differentiable.

With $f: \mathbb{R}^{m} \rightarrow \mathbb{R}$, we let $\operatorname{div}_{\alpha_{t}}(f)$ be the $\alpha_{t}$-divergence $m$-column vector operator with $j$-th entry given by the formula

$$
\operatorname{div}_{\alpha_{t}}(f)(x)^{j}:=\sum_{1 \leq i \leq m} \partial_{x_{i}}\left(\alpha_{t}^{i, j}(x) f(x)\right)
$$

The generator $L_{t}$ of the signal $X_{t}$ is also given by the second order differential operator

$$
L_{t}(f)(x):=\nabla f(x)^{\prime} b_{t}(x)+\frac{1}{2} \operatorname{Tr}\left(\nabla^{2} f(x) \alpha_{t}(x)\right) \quad \text { with the trace operator } \operatorname{Tr}(\cdot) .
$$

Here and throughout, and without further mention, we assume that functions $f$ acted on by a second-order differential generator are in addition twice differentiable with bounded derivatives.

For a measure $\mu$ and test function $f$ of compatible dimension we write,

$$
\mu(f):=\int \mu(d x) f(x)
$$

An integral operator $\mathcal{Q}(x, d z)$ acts on the right on scalar test functions $f$; and on the left on measures $\mu$ according to the formulae,

$$
\mathcal{Q}(f)(x):=\int \mathcal{Q}(x, d z) f(z) \text { and }(\mu \mathcal{Q})(d z):=\int \mu(d x) \mathcal{Q}(x, d z)
$$

We extend this operator to an integral operator on matrix functions $h(x)=\left(h_{i, j}(x)\right)_{i, j}$ by setting,

$$
\mathcal{Q}(h)(x)_{i, j}=\mathcal{Q}\left(h_{i, j}\right)(x)
$$

\section{Main Result}

In further development of this article we assume for any $t>0$ the conditional distribution $\pi_{t}$ has a positive density $p_{t}:=d \pi_{t} / d \lambda$ w.r.t. the Lebesgue measure $\lambda$ on $\mathbb{R}^{m}$. In addition, $p_{u}(x)$ and its derivative $\nabla p_{u}(x)$ are uniformly bounded w.r.t. $(u, x) \in[s, t] \times \mathbb{R}^{m}$, for any given $s>0$, almost surely w.r.t. the distribution of the observation process. A more detailed discussion on these regularity conditions is provided in Section 2.2 .

The main result of the article takes the following form: 
Theorem 2.1. For any $t \geq u \geq s$ we have the transport equation

$$
\pi_{t, s}(d x)=\left(\pi_{t, u} \mathcal{K}_{u, s}\right)(d x):=\int \pi_{t, u}(d z) \mathcal{K}_{u, s}(z, d x)
$$

where $\mathcal{K}_{u, s}$ denotes the Markov semigroup of the backward diffusion flow,

$$
d \mathcal{X}_{u, s}(x)=-\left(\left(p_{s}\left(\mathcal{X}_{u, s}(x)\right)^{-1} \operatorname{div}_{\alpha_{s}}\left(p_{s}\right)\left(\mathcal{X}_{u, s}(x)\right)-a_{s}\left(\mathcal{X}_{u, s}(x)\right)\right) d s+\sigma_{s}\left(\mathcal{X}_{u, s}(x)\right) d \mathcal{W}_{s}\right)
$$

with the boundary condition $\mathcal{X}_{u, u}(x)=x$, and where $\mathcal{W}_{t} \in \mathbb{R}^{p}$ denotes a p-dimensional Brownian motion independent of the observations.

The proof of the above theorem is provided in Section 4.1. The backward stochastic differential equation (2.2) should be read as shorthand for the backward Itô integration formula,

$$
\mathcal{X}_{t, s}(x)=x+\int_{s}^{t}\left(p_{u}\left(\mathcal{X}_{t, u}(x)\right)^{-1} \operatorname{div}_{\alpha_{u}}\left(p_{u}\right)\left(\mathcal{X}_{t, u}(x)\right)-a_{u}\left(\mathcal{X}_{t, u}(x)\right)\right) d u+\int_{s}^{t} \sigma_{u}\left(\mathcal{X}_{t, u}(x)\right) d \mathcal{W}_{u}
$$

with terminal condition $\mathcal{X}_{t, t}(x)=x$. The right-most term in the above formula is an Itô backward stochastic integral such that for any terminal time $t$ this process is a square integrable backward martingale w.r.t. the variable $s \in[0, t]$.

Formally, we may slice the time interval $[s, t]_{h}:=\left\{u_{0}, \ldots, u_{n-1}\right\}$ via some time mesh $u_{i+1}=$ $u_{i}+h$ from $u_{0}=s$ to $u_{n}=t$ and with time step $h>0$. In this notation, according to the backward equation (2.2), or (2.3), we compute $\mathcal{X}_{t, u-h}(x)$ from $\mathcal{X}_{t, u}(x)$ using the formula

$$
\mathcal{X}_{t, u-h}-\mathcal{X}_{t, u} \simeq\left(p_{u}\left(\mathcal{X}_{t, u}\right)^{-1} \operatorname{div}_{\alpha_{u}}\left(p_{u}\right)\left(\mathcal{X}_{t, u}\right)-a_{u}\left(\mathcal{X}_{t, u}\right)\right) h+\sigma_{u}\left(\mathcal{X}_{t, u}\right)\left(\mathcal{W}_{u}-\mathcal{W}_{u-h}\right)
$$

We may provide some comments on the above theorem. By construction, given the observations and for any given $x \in \mathbb{R}^{m}$ and $t \geq s$, the probability $\mathcal{K}_{t, s}(x, d z)$ introduced in (2.1) coincides with the distribution of the random state $\mathcal{X}_{t, s}(x)$. In addition, for any $t \geq u \geq s$ we have the integral and stochastic semigroup properties,

$$
\mathcal{K}_{t, s}\left(x_{2}, d x_{0}\right):=\int \mathcal{K}_{t, u}\left(x_{2}, d x_{1}\right) \mathcal{K}_{u, s}\left(x_{1}, d x_{0}\right)
$$

and

$$
\mathcal{X}_{t, s}=\mathcal{X}_{u, s} \circ \mathcal{X}_{t, u}
$$

where $\mathcal{X}_{u, s} \circ \mathcal{X}_{t, u}$ denotes the composition of the mappings $\mathcal{X}_{u, s}$ and $\mathcal{X}_{t, u}$.

If we let $\mathcal{X}_{t}$ be a random variable with distribution $\pi_{t}$, for some $t \geq 0$. According to (2.1) the random state $\mathcal{X}_{t, s}\left(\mathcal{X}_{t}\right)$ of the process (2.2) at any given $s \in[0, t]$, is distributed according to $\pi_{t, s}=\pi_{t} \mathcal{K}_{t, s}$. In words, the backward process $\mathcal{X}_{t, s}\left(\mathcal{X}_{t}\right)$ is distributed according to the smoothing distribution $\pi_{t, s}$ for any $s \leq t$ whenever the terminal condition $\mathcal{X}_{t, t}\left(\mathcal{X}_{t}\right)=\mathcal{X}_{t}$ is distributed according to the filtering distribution $\pi_{t}$. In this sense, (2.2) constitutes a backward nonlinear smoothing diffusion. A forward diffusion flow that has a marginal distribution at any time equal to the filtering distribution is considered in [42, 43].

More generally, we have the backward Itô formula

$$
d f\left(\mathcal{X}_{t, s}(x)\right)=-\mathcal{L}_{s, \pi_{s}}(f)\left(\mathcal{X}_{t, s}(x)\right) d s-\nabla f\left(\mathcal{X}_{t, s}(x)\right)^{\prime} \sigma_{s}\left(\mathcal{X}_{t, s}(x)\right) d \mathcal{W}_{s}
$$

with the second order differential operator

$$
\mathcal{L}_{s, \pi_{s}}(f)=\sum_{1 \leq j \leq m}\left(-a_{s}^{j}+\frac{1}{p_{s}} \operatorname{div}_{\alpha_{s}}\left(p_{s}\right)^{j}\right) \partial_{x_{j}} f+\frac{1}{2} \sum_{1 \leq i, j \leq m} \alpha_{s}^{i, j} \partial_{x_{i} x_{j}} f
$$


Equivalently, we have the backward martingale decomposition

$$
f\left(\mathcal{X}_{t, s}(x)\right)-f(x)-\int_{s}^{t} \mathcal{L}_{u, \pi_{u}}(f)\left(\mathcal{X}_{t, u}(x)\right) d u=\int_{s}^{t} \nabla f\left(\mathcal{X}_{t, u}(x)\right)^{\prime} \sigma_{u}\left(\mathcal{X}_{t, u}(x)\right) d \mathcal{W}_{u}
$$

This yields the backward evolution equations,

$$
\partial_{s} \mathcal{K}_{t, s}(f)(x)=-\mathcal{K}_{t, s}\left(\mathcal{L}_{s, \pi_{s}}(f)\right)(x)
$$

and

$$
\partial_{s} \pi_{t, s}(f)=-\pi_{t, s}\left(\mathcal{L}_{s, \pi_{s}}(f)\right)
$$

with terminal conditions $\mathcal{K}_{t, t}(f)=f$ and $\pi_{t, t}=\pi_{t}$. The formula (2.11) coincides with the conditional Fokker-Planck equation in [27, and further developed in [1].

For further discussion on general backward integration of stochastic flows see [10]; see also the appendix of [4] in the context of nonlinear diffusions, and [34] in the context of nonlinear filtering, and [13] on forward-backward perturbation analysis of stochastic flows. Note there is no issue with adaption of the backward process in the sense studied in [35] since we rely only on the independent backward Brownian motion in (2.2). The "backward diffusion" in (2.2) is backward in the sense of a time reversed stochastic differential equation as in [2, 14, 30].

\subsection{Some corollaries}

We end this introduction with some direct consequences of the above theorem.

Note when $b_{t}=0$ the measure $\pi_{t}$ coincides with the distribution of the random state $X_{t}$ of the signal. In this context, $\mathcal{X}_{t, s}\left(X_{t}\right)$ reduces to the time reversal of the signal starting at $\mathcal{X}_{t, t}\left(X_{t}\right)=X_{t}$ at the terminal time $t$. Using Theorem 2.1 we recover the fact that the time reversal process of the signal is itself a Markov diffusion [2, 14, 30]. More precisely, we have the corollary:

Corollary 2.2 (Anderson [2]). Assume that $b_{t}=0$. For any time horizon $t \geq 0$, the process $\mathfrak{X}_{s}^{t}:=X_{t-s}$ with $s \in[0, t]$ is a Markov process with generator

$$
\mathfrak{L}_{s}^{t}(f)=\sum_{1 \leq j \leq m}\left(\frac{1}{p_{t-s}} \operatorname{div}_{\alpha_{t-s}}\left(p_{t-s}\right)^{j}-a_{t-s}^{j}\right) \partial_{x_{j}} f+\frac{1}{2} \sum_{1 \leq i, j \leq m} \alpha_{t-s}^{i, j} \partial_{x_{i} x_{j}} f
$$

We consider now linear-Gaussian filtering/smoothing models with,

$$
a_{t}(x)=A_{t} x, \quad b_{t}(x)=B_{t} x \quad \text { and homogeneous diffusion matrix } \quad \sigma_{t}(x)=\Sigma_{t}
$$

for some matrices $\left(A_{t}, B_{t}, \Sigma_{t}\right)$ with appropriate dimensions. Whenever $X_{0}$ is a Gaussian random variable with mean $\widehat{X}_{0}$ and covariance matrix $R_{0}$, the optimal filter $\pi_{t}$ is a Gaussian distribution with mean $\widehat{X}_{t}$ and covariance matrix $R_{t}$ satisfying the Kalman-Bucy and the Riccati equations

$$
\left\{\begin{aligned}
d \widehat{X}_{t} & =A_{t} \widehat{X}_{t} d t+R_{t} B_{t}^{\prime} \beta_{t}^{-1}\left(d Y_{t}-B_{t} \widehat{X}_{t} d t\right) \\
\partial_{t} R_{t} & =A_{t} R_{t}+R_{t} A_{t}^{\prime}+\alpha_{t}-R_{t} B_{t}^{\prime} \beta_{t}^{-1} B_{t} R_{t}
\end{aligned}\right.
$$

In this context, we also have that

$$
-p_{s}(x)^{-1} \operatorname{div}_{\alpha_{s}}\left(p_{s}\right)(x)=\alpha_{s} R_{s}^{-1}\left(x-\widehat{X}_{s}\right)
$$

This yields the following corollary: 
Corollary 2.3. For linear Gaussian filtering models (2.13), the diffusion flow $\mathcal{X}_{t, s}(x)$ satisfies the backward evolution equation

$$
d \mathcal{X}_{t, s}(x)=-\left(\left(-A_{s} \mathcal{X}_{t, s}(x)-\alpha_{s} R_{s}^{-1}\left(\mathcal{X}_{t, s}(x)-\widehat{X}_{s}\right)\right) d s+\Sigma_{s} d \mathcal{W}_{s}\right)
$$

with the boundary condition $\mathcal{X}_{t, t}(x)=x$.

Replacing $x$ in (2.16) by a random variable $\mathcal{X}_{t}$ with distribution $\pi_{t}$ for any $t \geq s$ we have that $\mathcal{X}_{t, s}\left(\mathcal{X}_{t}\right)$ has distribution $\pi_{t, s}$. In addition, since the process is linear the distribution $\pi_{t, s}$ is Gaussian

with mean $\widehat{X}_{t, s}$ and covariance matrix $R_{t, s}$. The discrete time version of (2.16) can be found in Section 9.9.6 in [12].

Now taking expectations we readily deduce the rather well-known Rauch-Tung-Striebel smoothing equations [37], simplifying the innovation techniques and the sophisticated approximation theory developed in [16, 27, 39], or the formal variational approaches and maximum likelihood techniques presented in the pioneering articles [6, 37].

Corollary 2.4 (Rauch-Tung-Striebel [37]). For any $t \geq s$, the parameters $\left(\widehat{X}_{t, s}, R_{t, s}\right)$ satisfy the backward evolution equations

$$
\left\{\begin{array}{l}
\partial_{s} \widehat{X}_{t, s}=A_{s} \widehat{X}_{t, s}+\alpha_{s} P_{s}^{-1}\left(\widehat{X}_{t, s}-\widehat{X}_{s}\right) \\
\partial_{s} R_{t, s}=\left(A_{s}+\alpha_{s} R_{s}^{-1}\right) R_{t, s}+R_{t, s}\left(A_{s}+\alpha_{s} R_{s}^{-1}\right)^{\prime}-\alpha_{s}
\end{array}\right.
$$

with terminal conditions $\left(\widehat{X}_{t, t}, R_{t, t}\right)=\left(\widehat{X}_{t}, R_{t}\right)$.

\subsection{Comments on our regularity conditions}

We end this section with some comments on the regularity conditions discussed at the beginning of Section 2, These conditions are clearly met for linear Gaussian filtering models (see e.g. (2.14) and (2.15) $)$. They are also met for nonlinear models as soon as the signal satisfies a classical controllability-type condition.

Note firstly, whenever the signal is uniformly elliptic, in the sense that $\alpha_{t}(x)=\sigma_{t}(x) \sigma_{t}^{\prime}(x) \geq \delta I$ for some $\delta>0$, then it is well known that $X_{t}$ has a smooth positive density w.r.t. the Lebesgue measure on $\mathbb{R}^{m}$. Nevertheless in many important applications this ellipticity condition is not satisfied. The parabolic Hörmander condition for time varying models [7, 15] is a weaker condition. For linear-Gaussian filtering problems, this condition reduces to the usual controllability condition. Indeed, if we replace the Brownian motions $W_{t}$ by some arbitrary smooth control functions, all states are accessible from one to another, as soon as the Lie algebra generated by the controlled vector fields is of full rank. This result is also called the Chow-Rashevskii theorem [8, 38]. Under this Hörmander condition, the Hörmander theorem [15] ensures that the signal states have a smooth density w.r.t. the Lebesgue measure on $\mathbb{R}^{m}$. In addition, for any $s<t$ the Markov transition semigroup $P_{s, t}$ of the signal has a smooth positive density $(x, z) \mapsto p_{s, t}(x, z)$ w.r.t. the Lebesgue measure $\lambda$ on $\mathbb{R}^{m}$. In addition, the integral operator $P_{s, t}$ with $s<t$ maps test functions $f$ into bounded smooth functions $P_{s, t}(f)$ given by

$$
P_{s, t}(f)(x)=\int P_{s, t}(x, d z) f(z)=\int f(z) p_{s, t}(x, z) d z
$$

A natural way to transfer the smoothing properties of $P_{s, t}$ to the optimal filter is to use the following equation

$$
\pi_{t}(f)=\pi_{0}\left(P_{0, t}(f)\right)+\int_{0}^{t} \pi_{s}\left(P_{s, t}(f)\left(b_{s}-\pi_{s}\left(b_{s}\right)\right)\right)^{\prime} \beta_{s}^{-1}\left(d Y_{s}-\pi_{s}\left(b_{s}\right) d s\right)
$$


given in Theorem 1.1 in [22]. Using this formula we readily check that for any $t>0$ the conditional distribution $\pi_{t}$ has a positive density $p_{t}$ on $\mathbb{R}^{m}$. Whenever $\sigma_{t}(x)$ and $b_{t}(x)$ are also bounded, Theorem 3.6 in [29] (see also Theorem 6.3 in [23]) also ensures that $p_{u}$ is smooth, and for any $k \geq 1$, any parameters $h>0$ and any time horizon $t>0$ we have

$$
\sup _{h \leq s \leq t} \sup _{x \in \mathbb{R}^{m}}\left(\left|p_{s}(x)\right|+\left\|\nabla^{k} p_{s}(x)\right\|\right)<\infty
$$

where $\|\cdot\|$ stands for any (equivalent) norm on $\mathbb{R}^{m}$.

The above estimates are met for linear Gaussian filtering models. Nevertheless, some caution must be used when considering estimates of the form (2.19). Indeed, most of the literature on stochastic partial differential equations arising in nonlinear filtering, such as the strong formulation of the Zakai and Kushner-Stratonovich equations, assume that the sensor function is uniformly bounded, see e.g. [23, 29, 34] and [41, 44]. To the best of our knowledge the extension of the estimate (2.19) to more general unbounded sensor functions is still an open important question.

We also note here that the Kallianpur-Striebel formula [17, 18] is valid as soon as $\beta_{u} \geq \epsilon I$, for some $\epsilon>0$ and the functions $\left(a_{u}(x), b_{u}(x), \sigma_{u}(x)\right)$ are smooth with uniformly bounded derivatives w.r.t $x$ of all order on $(u, x) \in[s, t] \times \mathbb{R}^{m}$, for any $s \leq t$. Weaker conditions can also be found in the book [5] and the recent article [9].

Since $X_{t}$ has continuous paths, for any continuous function $f$ and any $s \leq t$ the random mapping $u \in[s, t] \mapsto f\left(X_{u}\right)$ is almost surely a uniformly bounded function. In addition, $f\left(X_{t}\right)$ is integrable as soon as $f$ has polynomial growth. Up to some classical localization procedure (see e.g. Chapter 7 in [40]), these rather weak regularity properties also ensure that the integral semigroups that transport (in time) the filtering measures discussed in Section 3, as well as their stochastic partial differential evolution equations, are well defined on any test function with polynomial growth.

\section{Nonlinear filtering equations}

As is well known (e.g. see [6, 37, 16, 39, 27, 1, 3, 34]), a solution to the smoothing problem will typically make use of the solution of a related filtering in some way. Consequently, we need to present and develop some related filtering results for proving our main result, Theorem 2.1. This section is largely self-contained but it is vital in the proof, in Section 4, of our main result.

The first part of this section presents the classical Kallianpur-Striebel formula which acts as a continuous-time version of Bayes law. In Sections 3.1 and 3.2 respectively we present the Zakai, and Kushner-Stratonovich equations for the flow of the conditional filtering distributions (both unnormalised and normalised). These results are rather well known. For further background on these classical ideas, we refer to the pioneering articles by Kallianpur and Striebel [17, 18, and by Kushner [26] and Zakai [44]. For more recent discussion on these probabilistic models, we refer to [9], and [5, 12, and the references therein. In this article, we present a novel and self contained derivation based on stochastic transport semigroups and their forward evolution equations.

The solution of the Zakai equation is sometimes termed the unnormalized filter. The semigroup that transports these filtering measures (in time) is discussed in Section 3.1, and its normalized version in Section 3.2. Section 3.3 presents a novel direct approach for deriving the backward evolution of these transport semigroups. Our approach in Section 3.3 combines the backward Itô formula for stochastic flows with the backward Itô-Ventzell formula presented in [13].

Now, we introduce some notation/terminology and briefly present the Kallianpur-Striebel formula and the linear semigroup property of unnormalized measures. Let $X_{s, t}(x)$ be the stochastic 
flow of the signal on the time interval $[s, t]$ and starting at $x$ at time $s$. Let $Z_{s, t}(x)$ be the multiplicative functional

$$
\log Z_{s, t}(x):=\int_{s}^{t} b_{u}\left(X_{s, u}(x)\right)^{\prime} \beta_{u}^{-1} d Y_{u}-\frac{1}{2} \int_{s}^{t} b_{u}\left(X_{s, u}(x)\right)^{\prime} \beta_{u}^{-1} b_{u}\left(X_{s, u}(x)\right) d u
$$

When $x$ is replaced by $X_{s}$ we may write $Z_{s, t}$ instead of $Z_{s, t}\left(X_{s}\right)$, and when $s=0$, we may also write $Z_{t}$ instead of $Z_{0, t}$. With this notation, we have the classical Kallianpur-Striebel formula,

$$
\pi_{t}(f)=\gamma_{t}(f) / \gamma_{t}(1) \quad \text { with } \quad \gamma_{t}(f):=\mathbb{E}_{0}\left(f\left(X_{t}\right) Z_{t}\right)
$$

Here, $\mathbb{E}_{0}(\cdot)$ denotes the expectation operator w.r.t. the signal with a fixed observation process.

The transport semigroup of the unnormalized measures $\gamma_{t}$ is given for any $s \leq t$ by the formula

$$
\gamma_{t}=\gamma_{s} Q_{s, t} \quad \text { with } \quad Q_{s, t}(f)(x):=\mathbb{E}_{0}\left(f\left(X_{s, t}(x)\right) Z_{s, t}(x)\right)
$$

To check this claim observe that,

$$
Z_{t}=Z_{s} Z_{s, t} \quad \Longrightarrow \quad \mathbb{E}_{0}\left(f\left(X_{t}\right) Z_{t}\right)=\mathbb{E}_{0}\left(Z_{s} \mathbb{E}_{0}\left(f\left(X_{t}\right) Z_{s, t} \mid X_{s}\right)\right)=\mathbb{E}_{0}\left(Z_{s} Q_{s, t}(f)\left(X_{s}\right)\right)
$$

Now for any $s \leq u \leq t$ we have

$$
\begin{aligned}
Q_{s, t}(f)\left(X_{s}\right)=\mathbb{E}_{0}\left(f\left(X_{t}\right) Z_{s, t} \mid X_{s}\right) & =\mathbb{E}_{0}\left(Z_{s, u} \mathbb{E}\left(f\left(X_{t}\right) Z_{u, t} \mid X_{u}\right) \mid X_{s}\right) \\
& =\mathbb{E}_{0}\left(Z_{s, u} Q_{u, t}(f)\left(X_{u}\right) \mid X_{s}\right)=Q_{s, u}\left(Q_{u, t}(f)\right)\left(X_{u}\right)
\end{aligned}
$$

This yields the integral semigroup formula

$$
Q_{s, t}\left(x_{0}, d x_{2}\right)=\left(Q_{s, u} Q_{u, t}\right)\left(x_{0}, d x_{2}\right):=\int Q_{s, u}\left(x_{0}, d x_{1}\right) Q_{s, u}\left(x_{1}, d x_{2}\right)
$$

In a more compact form, the semigroup property takes the form

$$
Q_{s, t}=Q_{s, u} Q_{u, t} \quad \text { with } \quad Q_{t, t}=I \quad \text { where } I \text { denotes the identity operator. }
$$

\subsection{Unnormalized stochastic semigroups}

Consider the stochastic transport semigroups $\mathbb{P}_{s, t}$ and $\mathbb{Q}_{s, t}$ defined by the composition of functions

$$
\mathbb{P}_{s, t}(f)(x):=\left(f \circ X_{s, t}\right)(x) \text { and } \mathbb{Q}_{s, t}(f)(x):=\mathbb{P}_{s, t}(f)(x) Z_{s, t}(x)
$$

Using the semigroup properties of the stochastic flow $X_{s, t}(x)$ for any $s \leq u \leq t$ we check that

$$
\mathbb{P}_{s, t}(f)(x)=\left(f \circ X_{s, t}\right)(x)=\left(f \circ X_{u, t}\right)\left(X_{s, u}(x)\right)=\mathbb{P}_{s, u}\left(\mathbb{P}_{u, t}(f)\right)(x)
$$

Similarly, we have

$$
\mathbb{Q}_{s, t}(f)(x)=Z_{s, u}(x)\left(Z_{u, t}\left(X_{s, u}(x)\right)\left(f \circ X_{s, t}\right)\left(X_{s, u}(x)\right)\right)=\mathbb{Q}_{s, u}\left(\mathbb{Q}_{u, t}(f)\right)(x)
$$

In a more compact form we have the semigroup properties

$$
\mathbb{P}_{s, t}=\mathbb{P}_{s, u} \circ \mathbb{P}_{u, t} \quad \text { and } \quad \mathbb{Q}_{s, t}=\mathbb{Q}_{s, u} \circ \mathbb{Q}_{u, t} \quad \text { with } \quad \mathbb{P}_{t, t}=I=\mathbb{Q}_{t, t}
$$

Also observe that

$$
P_{s, t}(f)(x):=\mathbb{E}_{0}\left(\mathbb{P}_{s, t}(f)(x)\right) \quad \text { and } \quad Q_{s, t}(f)(x):=\mathbb{E}_{0}\left(\mathbb{Q}_{s, t}(f)(x)\right)
$$

The forward evolution equations of the above semigroups are described in the next proposition. 
Proposition 3.1. For any $t \geq s$ we have the forward stochastic evolution equation

$$
d \mathbb{Q}_{s, t}(f)=\mathbb{Q}_{s, t}\left(L_{t}(f)\right) d t+\mathbb{Q}_{s, t}\left(f b_{t}^{\prime}\right) \beta_{t}^{-1} d Y_{t}+\mathbb{Q}_{s, t}\left((\nabla f)^{\prime} \sigma_{t}\right) d W_{t}
$$

with initial condition $\mathbb{Q}_{s, s}(f)=f$, when $t=s$. In particular, we have the forward equation

$$
d Q_{s, t}(f)=Q_{s, t}\left(L_{t}(f)\right) d t+Q_{s, t}\left(f b_{t}^{\prime}\right) \beta_{t}^{-1} d Y_{t}
$$

with the initial condition $Q_{s, s}(f)=f$, when $t=s$.

Proof. Assume that the sensor function $b_{u}(x)$ is uniformly bounded on $[s, t] \times \mathbb{R}^{m}$, for any $s \leq t$. In this situation, the random process $\left(X_{s, u}(x), Z_{s, u}(x)\right)$ also has uniformly bounded absolute moments of any order on any compact interval $[s, t]$, for any time parameters $s \leq t$. In this context, we use Itô formula to check that

$$
d Z_{s, t}(x)=Z_{s, t}(x) b_{t}\left(X_{s, t}(x)\right)^{\prime} \beta_{t}^{-1} d Y_{t}
$$

as well as

$$
d \mathbb{P}_{s, t}(f)(x)=\mathbb{P}_{s, t}\left(L_{t}(f)\right)(x) d t+\mathbb{P}_{s, t}\left(\nabla f^{\prime} \sigma_{t}\right)(x) d W_{t}
$$

An integration by parts yields

$$
\begin{aligned}
d \mathbb{Q}_{s, t}(f)(x)= & Z_{s, t}(x) d \mathbb{P}_{s, t}(f)(x)+\mathbb{P}_{s, t}(f)(x) d Z_{s, t}(x) \\
= & \left(L_{t}(f)\left(X_{s, t}(x)\right) Z_{s, t}(x) d t+Z_{s, t}(x) f\left(X_{s, t}(x)\right) b_{t}\left(X_{s, t}(x)\right)^{\prime} \beta_{t}^{-1} d Y_{t}\right) \\
& \quad+Z_{s, t}(x) \nabla f\left(X_{s, t}(x)\right)^{\prime} \sigma_{t}\left(X_{s, t}(x)\right) d W_{t}
\end{aligned}
$$

By classical localization principles of Itô integrals (see for instance Chapter 7 in [40]), the above result is also true for unbounded sensor functions. This ends the proof of (3.3). Taking the expectations, we conclude that

$$
d \mathbb{E}_{0}\left(\mathbb{Q}_{s, t}(f)(x)\right)=\mathbb{E}_{0}\left(\mathbb{Q}_{s, t}\left(L_{t}(f)\right)(x)\right) d t+\mathbb{E}_{0}\left(\mathbb{Q}_{s, t}\left(f \quad b_{t}^{\prime}\right)(x)\right) \beta_{t}^{-1} d Y_{t}
$$

This ends the proof of (3.4). The proof of the proposition is completed.

Combining (3.2) with Fubini's theorem, we readily check the weak form of Zakai equation given by the formula

$$
d \gamma_{t}(f)=\gamma_{t}\left(L_{t}(f)\right) d t+\gamma_{t}\left(f b_{t}^{\prime}\right) \beta_{t}^{-1} d Y_{t}
$$

Arguing as in (2.18), we transfer the smoothing properties of $P_{s, t}$ to $Q_{s, t}$ using the perturbation formulae given for any $s<t$ by

$$
Q_{s, t}(f)=P_{s, t}(f)+\int_{s}^{t} Q_{s, u}\left(P_{u, t}(f) b_{u}^{\prime}\right) \beta_{u}^{-1} d Y_{u}
$$

Arguing as in [44, the above formula shows that for any $s<t$ the integral operator $Q_{s, t}\left(x_{0}, d x_{1}\right)$ has a density $x_{1} \mapsto q_{s, t}\left(x_{0}, x_{1}\right)$ w.r.t. the Lebesgue measure on $\mathbb{R}^{m}$ given by the integral equation

$$
q_{s, t}\left(x_{0}, x_{1}\right)=p_{s, t}\left(x_{0}, x_{1}\right)+\int_{s}^{t}\left[\int q_{s, u}\left(x_{0}, z\right) p_{u, t}\left(z, x_{1}\right) b_{u}^{\prime}(z) d z\right] \beta_{u}^{-1} d Y_{u}
$$




\subsection{Normalized stochastic semigroups}

Let $\bar{Z}_{s, t}(x)$ be the multiplicative functional defined as $Z_{s, t}(x)$ by replacing in (3.1) the function $b_{u}$ and the observation increment $d Y_{u}$ by the centered function $\bar{b}_{u}$ and the innovation increment $d \bar{Y}_{u}$ defined by the formulae

$$
\bar{b}_{u}:=b_{u}-\pi_{u}\left(b_{u}\right) \quad \text { and } \quad d \bar{Y}_{u}:=d Y_{u}-\pi_{u}\left(b_{u}\right) d u
$$

Under our assumptions, the random process $\pi_{t}\left(b_{t}\right)$ is almost surely square integrable on any compact time interval so that the innovation process is well defined. Choosing $f=1$ in (3.5) we check that

$$
\log \gamma_{t}(1)=\int_{0}^{t} \pi_{u}\left(b_{u}\right)^{\prime} \beta_{u}^{-1} d Y_{u}-\frac{1}{2} \int_{0}^{t} \pi_{u}\left(b_{u}\right)^{\prime} \beta_{u}^{-1} \pi_{u}\left(b_{u}\right) d u
$$

Observe that

$$
\pi_{s} Q_{s, t}(1)=\gamma_{t}(1) / \gamma_{s}(1)=\exp \left(\int_{s}^{t} \pi_{u}\left(b_{u}\right)^{\prime} \beta_{u}^{-1} d Y_{u}-\frac{1}{2} \int_{s}^{t} \pi_{u}\left(b_{u}\right)^{\prime} \beta_{u}^{-1} \pi_{u}\left(b_{u}\right) d u\right)
$$

We also consider the normalized stochastic semigroup

$$
\overline{\mathbb{Q}}_{s, t}(f)(x):=\left(f \circ X_{s, t}\right)(x) \bar{Z}_{s, t}(x)=\mathbb{P}_{s, t}(f)(x) \bar{Z}_{s, t}(x)
$$

Arguing as above, for any $s \leq u \leq t$ we check that

$$
\overline{\mathbb{Q}}_{s, t}=\overline{\mathbb{Q}}_{s, u} \circ \overline{\mathbb{Q}}_{u, t} \quad \text { and } \quad \bar{Z}_{s, t}(x)=Z_{s, t}(x) / \pi_{s} Q_{s, t}(1)
$$

Consider the semigroup

$$
\bar{Q}_{s, t}(f)(x):=\mathbb{E}_{0}\left(\overline{\mathbb{Q}}_{s, t}(f)(x)\right)=\mathbb{E}_{0}\left(f\left(X_{s, t}(x)\right) \bar{Z}_{s, t}(x)\right)=Q_{s, t}(f)(x) / \pi_{s} Q_{s, t}(1)
$$

In this notation, using the same arguments as in the proof of Proposition 3.1 we have the following forward evolution equations.

Proposition 3.2. For any given time horizon $s$ and for any $t \geq s$ we have the forward stochastic evolution equation

$$
d \overline{\mathbb{Q}}_{s, t}(f)=\overline{\mathbb{Q}}_{s, t}\left(L_{t}(f)\right) d t+\overline{\mathbb{Q}}_{s, t}\left(f \bar{b}_{t}^{\prime}\right) \beta_{t}^{-1} d \bar{Y}_{t}+\overline{\mathbb{Q}}_{s, t}\left((\nabla f)^{\prime} \sigma_{t}\right) d W_{t}
$$

with initial condition $\bar{Q}_{s, s}(f)=f$, when $t=s$. In particular, we have the forward equation

$$
d \bar{Q}_{s, t}(f)=\bar{Q}_{s, t}\left(L_{t}(f)\right) d t+\bar{Q}_{s, t}\left(f \bar{b}_{t}^{\prime}\right) \beta_{t}^{-1} d \bar{Y}_{t}
$$

with the initial condition $\bar{Q}_{s, s}(f)=f$, when $t=s$.

The above proposition yields the weak form of the Kushner-Stratonovich equation defined by

$$
d \pi_{t}(f)=\pi_{t}\left(L_{t}(f)\right) d t+\pi_{t}\left(f \bar{b}_{t}\right)^{\prime} \beta_{t}^{-1} d \bar{Y}_{t}
$$

Formally, using the same notation as in (3.11) we have the forward approximation equation

$$
\pi_{u+h}(f) \simeq \pi_{u}(f)+\pi_{u}\left(L_{u}(f)\right) h+\pi_{u}\left(f \bar{b}_{u}\right)^{\prime} \beta_{u}^{-1}\left(\bar{Y}_{u+h}-\bar{Y}_{u}\right)
$$




\subsection{Backward evolution equations}

This section is concerned with the backward evolution equation associated with the unnormalized semigroup $\mathbb{Q}_{s, t}$ and its normalized version. The main result of this section is the following theorem:

Theorem 3.3. For any twice differentiable function $f$ with bounded derivatives and for any $s \leq t$ we have the backward evolution equation

$$
\begin{array}{r}
d \mathbb{Q}_{s, t}(f)(x)=-\left(\nabla \mathbb{Q}_{s, t}(f)(x)^{\prime} a_{s}(x)+\frac{1}{2} \operatorname{Tr}\left(\nabla^{2} \mathbb{Q}_{s, t}(f)(x) \alpha_{s}(x)\right)\right) d s \\
-\mathbb{Q}_{s, t}(f)(x) b_{s}(x)^{\prime} \beta_{s}^{-1} d Y_{s}-\nabla \mathbb{Q}_{s, t}(f)(x)^{\prime} \sigma_{s}(x) d W_{s}
\end{array}
$$

with terminal condition $\mathbb{Q}_{t, t}(f)=f$, when $s=t$. In particular, we have the backward equation

$$
d Q_{s, t}(f)=-\left(L_{s}\left(Q_{s, t}(f)\right) d s+Q_{s, t}(f) b_{s}^{\prime} \beta_{s}^{-1} d Y_{s}\right)
$$

with terminal condition $Q_{t, t}(f)=f$, when $s=t$.

Proof. We use a direct approach combining the backward filtering calculus developed in [23, 41] based on the backward Itô calculus developed in [10, 11, 21, 25], see also the more recent article [13] and references therein.

Consider the stochastic flow $\chi_{s, t}(\bar{x})$ starting at

$$
\chi_{s, s}(\bar{x})=\bar{x}:=\left(\begin{array}{l}
x \\
z
\end{array}\right) \in\left(\mathbb{R}^{m} \times \mathbb{R}\right)
$$

on the time interval $[s, \infty[$ and given for any $t \geq s$ by

$$
\chi_{s, t}(\bar{x}):=\left(\begin{array}{c}
X_{s, t}(x) \\
Z_{s, t}(x) z
\end{array}\right) \in\left(\mathbb{R}^{m} \times \mathbb{R}\right)
$$

We set

$$
\begin{array}{rlrl}
\mathcal{B}_{t}(\bar{x}) & :=\left(\begin{array}{c}
a_{t}(x) \\
0
\end{array}\right) & \mathcal{U}_{t} & :=\left(\begin{array}{c}
W_{t} \\
Y_{t}
\end{array}\right) \\
\Lambda_{t}(\bar{x}) & :=\left(\begin{array}{cc}
\sigma_{t}(x) & 0 \\
0 & z b_{t}(x)^{\prime} \beta_{t}^{-1}
\end{array}\right) \text { and } \quad \mathcal{A}_{t}(\bar{x}) & :=\Lambda_{t}(\bar{x}) \Lambda_{t}(\bar{x})^{\prime}
\end{array}
$$

Assume that the sensor function $b_{u}(x)$ is uniformly bounded on $[s, t] \times \mathbb{R}^{m}$, for any $s \leq t$. Then, the process $\left(Z_{s, u}(x), \chi_{s, u}(\bar{x})\right)$ has continuous partial derivatives and also has uniformly bounded absolute moments of any order on $\left([s, t] \times \mathbb{R}^{m}\right)$, for any $s \leq t$. In this situation, we have the forward stochastic evolution equation

$$
d \chi_{s, t}(\bar{x})=\mathcal{B}_{t}\left(\chi_{s, t}(\bar{x})\right) d t+\Lambda_{t}\left(\chi_{s, t}(\bar{x})\right) d \mathcal{U}_{t}
$$

For any twice differentiable function $F$ on $\left(\mathbb{R}^{m} \times \mathbb{R}\right)$ with bounded derivatives we also have the backward equation

$$
\begin{aligned}
& d\left(F \circ \chi_{s, t}\right)(\bar{x}) \\
& =-\left(\nabla\left(F \circ \chi_{s, t}\right)(\bar{x})^{\prime} \mathcal{B}_{s}(\bar{x})+\frac{1}{2} \operatorname{Tr}\left(\nabla^{2}\left(F \circ \chi_{s, t}\right)(\bar{x}) \mathcal{A}_{s}(\bar{x})\right)\right) d s-\nabla\left(F \circ \chi_{s, t}\right)(\bar{x})^{\prime} \Lambda_{s}(\bar{x}) d \mathcal{U}_{s}
\end{aligned}
$$


A proof of the above formula can be found in the articles [10, 11], see also [13]. Choosing the function $F(\bar{x})=f(x) z$, for some twice differentiable function $f$ on $\mathbb{R}^{m}$ with bounded derivatives and letting $z=1$ we check that

$$
\begin{aligned}
& d\left(f\left(X_{s, t}(x)\right) Z_{s, t}(x)\right) \\
& =-\left(\nabla\left(f\left(X_{s, t}(x)\right) Z_{s, t}(x)\right)^{\prime} a_{s}(x)+\frac{1}{2} \operatorname{Tr}\left(\nabla^{2}\left(f\left(X_{s, t}(x)\right) Z_{s, t}(x)\right) \alpha_{s}(x)\right)\right) d s \\
& \quad-\left(f\left(X_{s, t}(x)\right) Z_{s, t}(x)\right) b_{s}(x)^{\prime} \beta_{s}^{-1} d Y_{s}-\nabla\left(f\left(X_{s, t}(x)\right) Z_{s, t}(x)\right)^{\prime} \sigma_{s}(x) d W_{s}
\end{aligned}
$$

This ends the proof of (3.9). By localization arguments, the above result is also true for unbounded sensor functions. Integrating the flow of the signal we obtain (3.10). This ends the proof of the theorem.

We can also check (3.10) considering a discrete time interval $[s, t]_{h}:=\left\{t_{0}, \ldots, t_{n-1}\right\}$ associated with some refining time mesh $t_{i+1}=t_{i}+h$ from $t_{0}=s$ to $t_{n}=t$, for some time step $h>0$. By (3.4), for any $u \in[s, t]_{h}$ we compute $Q_{u, t}(f)$ from $Q_{u+h, t}(f)$ using the backward equation

$$
\begin{aligned}
Q_{u, t}(f) & =Q_{u+h, t}(f)+\left(Q_{u, u+h}-I\right)\left(Q_{u+h, t}(f)\right) \\
& \simeq Q_{u+h, t}(f)+L_{u}\left(Q_{u+h, t}(f)\right) h+Q_{u+h, t}(f) b_{u}^{\prime} \beta_{u}^{-1}\left(Y_{u+h}-Y_{u}\right)
\end{aligned}
$$

For null sensor functions the evolution equation (3.9) coincides with the backward Itô formula discussed in [10, 11, 13, 23, 24].

Choosing $f=1$ in (3.10) we recover the backward evolution of the likelihood function presented in [3, 33] (see formula (5.9) in [3] and equation (3.15) in [33]). Arguing as in (3.6), using (3.10) we check the perturbation formulae given for any $s<t$ by,

$$
Q_{s, t}(f)=P_{s, t}(f)+\int_{s}^{t} P_{s, u}\left(Q_{u, t}(f) b_{u}^{\prime}\right) \beta_{u}^{-1} d Y_{u}
$$

Thus, for any $s<t$ the integral operator $Q_{s, t}\left(x_{0}, d x_{1}\right)$ has a density $\left(x_{0}, x_{1}\right) \mapsto q_{s, t}\left(x_{0}, x_{1}\right)$ given by (3.6) and the integral formula,

$$
q_{s, t}\left(x_{0}, x_{1}\right)=p_{s, t}\left(x_{0}, x_{1}\right)+\int_{s}^{t}\left[\int p_{s, u}\left(x_{0}, z\right) q_{u, t}\left(z, x_{1}\right) b_{u}^{\prime}(z) d z\right] \beta_{u}^{-1} d Y_{u}
$$

Using the same arguments as in the proof of Theorem 3.3 we also have the following backward evolution equation.

Proposition 3.4. For any twice differentiable function $f$ with bounded derivatives and for any $s \leq t$ we also have the backward equation

$$
\begin{aligned}
d \overline{\mathbb{Q}}_{s, t}(f)(x)=-\left(\nabla \overline{\mathbb{Q}}_{s, t}(f)(x)^{\prime} a_{s}(x)+\frac{1}{2} \operatorname{Tr}\left(\nabla^{2} \overline{\mathbb{Q}}_{s, t}(f)(x) \alpha_{s}(x)\right)\right) d s \\
-\overline{\mathbb{Q}}_{s, t}(f)(x) \bar{b}_{s}(x)^{\prime} \beta_{s}^{-1} d \bar{Y}_{s}-\nabla \overline{\mathbb{Q}}_{s, t}(f)(x)^{\prime} \sigma_{s}(x) d W_{s}
\end{aligned}
$$

with terminal condition $\overline{\mathbb{Q}}_{t, t}(f)=f$. In particular, we have the backward equation,

$$
d \bar{Q}_{s, t}(f)=-\left(L_{s}\left(\bar{Q}_{s, t}(f)\right) d s+\bar{Q}_{s, t}(f) \bar{b}_{s}^{\prime} \beta_{s}^{-1} d \bar{Y}_{s}\right)
$$

with terminal condition $\bar{Q}_{t, t}(f)=f$.

Using the same notation as in (3.11), we also have the approximating backward equation

$$
\bar{Q}_{u, t}(f) \simeq \bar{Q}_{u+h, t}(f)+L_{u}\left(\bar{Q}_{u+h, t}(f)\right) h+\bar{Q}_{u+h, t}(f) \bar{b}_{u}^{\prime} \beta_{u}^{-1}\left(\bar{Y}_{u+h}-\bar{Y}_{u}\right)
$$




\section{Smoothing semigroups and proof of the main result}

This section is concerned with forward-backward evolution equations for the conditional smoothing distribution and the proof of our main result.

Let $\mathcal{K}_{t, s}$ be the backward integral operator defined by,

$$
\mathcal{K}_{t, s}(f)(x):=\int \pi_{s}(d z) \frac{d \bar{Q}_{s, t}(z, \cdot)}{d \pi_{t}}(x) f(z)
$$

For any $s \leq u \leq t$ we have the backward semigroup property,

$$
\mathcal{K}_{t, s}=\mathcal{K}_{t, u} \mathcal{K}_{u, s}
$$

which follows via,

$$
\begin{aligned}
\left(\mathcal{K}_{t, u} \mathcal{K}_{u, s}\right)(f)(x) & =\int \pi_{s}\left(d x_{0}\right) \bar{Q}_{s, u}\left(x_{0}, d x_{1}\right) \frac{d \bar{Q}_{u, t}\left(x_{1}, \cdot\right)}{d \pi_{t}}(x) f\left(x_{0}\right) \\
& =\int \pi_{s}\left(d x_{0}\right) \frac{d \bar{Q}_{s, t}\left(x_{0}, \cdot\right)}{d \pi_{t}}(x) f\left(x_{0}\right)=\mathcal{K}_{t, s}(f)(x)
\end{aligned}
$$

and where we exploit the semigroup properties of the operators $\bar{Q}_{s, t}$.

Also observe that for any $t>s>0$ the integral operator $\mathcal{K}_{t, s}\left(x_{1}, d x_{0}\right)$ has a density $\left(x_{1}, x_{0}\right) \mapsto$ $k_{s, t}\left(x_{1}, x_{0}\right)$ w.r.t. the Lebesgue measure on $\mathbb{R}^{m}$ given by,

$$
k_{t, s}\left(x_{1}, x_{0}\right):=p_{s}\left(x_{0}\right) \bar{q}_{s, t}\left(x_{0}, x_{1}\right) / p_{t}\left(x_{1}\right) \quad \text { with } \quad \bar{q}_{s, t}\left(x_{0}, x_{1}\right)=q_{s, t}\left(x_{0}, x_{1}\right) / \pi_{s}\left(Q_{s, t}(1)\right)
$$

The function $q_{s, t}$ denotes the density of the integral operator $Q_{s, t}$ discussed in (3.6) and (3.12).

Now, for any pair of functions $(f, g)$ we readily check the duality formula,

$$
\pi_{s}\left(f \bar{Q}_{s, t}(g)\right)=\pi_{t}\left(\mathcal{K}_{t, s}(f) g\right)
$$

The following technical result is key in the proof of Theorem 2.1 .

Lemma 4.1. For any time parameter $s \leq t$ we have the forward-backward differential equation

$$
\partial_{s}\left(\pi_{s}\left(f \bar{Q}_{s, t}(g)\right)\right)=-\pi_{s}\left(\bar{Q}_{s, t}(g) \mathcal{L}_{s, \pi_{s}}(f)\right)
$$

with the second order differential operator

$$
\mathcal{L}_{s, \pi_{s}}(f):=-L_{s}(f)+\frac{1}{p_{s}} \sum_{1 \leq i, j \leq m} \partial_{x_{i}}\left(p_{s} \alpha_{s}^{i, j} \partial_{x_{j}} f\right)
$$

Proof. Observe that (4.4) does not involve the derivatives of the function $g$. Thus, up to a smooth mollifier's type approximation of the function $g$, it suffices to check (4.4) for any pair of bounded and twice differentiable functions $f, g$ with bounded differentials. Arguing as in the proof of Proposition 3.1 and Theorem 3.3, it suffices to prove the result for uniformly bounded sensor functions $b_{u}(x)$ on $[s, t] \times \mathbb{R}^{m}$, for any $s \leq t$.

In this situation, for any time horizon $t$, combining the Kushner-Stratonovich equation (3.7) with the backward equation (3.13) for any $s \leq t$, we check the forward-backward evolution equation

$$
\partial_{s}\left(\pi_{s}\left(f \bar{Q}_{s, t}(g)\right)\right)=\pi_{s}\left(L_{s}\left(f \bar{Q}_{s, t}(g)\right)-f L_{s}\left(\bar{Q}_{s, t}(g)\right)\right)
$$


The above equation can be proved using the backward Itô-Ventzell formula in [13. We use the same notation as in the proof of Theorem 3.3 . Let $\bar{Z}_{s, t}(x)$ be the multiplicative functional defined as $Z_{s, t}(x)$ by replacing the function $b_{u}$ and the observation Itô-increment $d Y_{u}$ by the centered function $\bar{b}_{u}$ and the innovation increment $d \bar{Y}_{u}$.

Consider the backward random field $F_{s, t}$ with terminal condition $F_{t, t}(\bar{x})=f(x) g(x) z$ defined by the formula

$$
F_{s, t}(\bar{x}):=f(x) \overline{\mathbb{Q}}_{s, t}(g)(x) z \quad \text { and we set } \quad \bar{\chi}_{s}:=\left(\begin{array}{c}
X_{s} \\
\bar{Z}_{s}
\end{array}\right) \in\left(\mathbb{R}^{m} \times \mathbb{R}\right) .
$$

In this notation, we have

$$
\mathbb{E}_{0}\left(F_{s, t}\left(\bar{\chi}_{s}\right)\right)=\mathbb{E}_{0}\left(f\left(X_{s}\right) \bar{Z}_{s} \mathbb{E}_{0}\left(\overline{\mathbb{Q}}_{s, t}(g)\left(X_{s}\right) \mid\left(X_{s}, Z_{s}\right)\right)\right)=\pi_{s}\left(f \bar{Q}_{s, t}(g)\right)
$$

Observe that

$$
F_{s, t}(\bar{x})=f(x)\left(F \circ \bar{\chi}_{s, t}\right)(\bar{x})
$$

with the function

$$
F(\bar{x}):=g(x) z \quad \text { and the stochastic flow } \quad \bar{\chi}_{s, t}(x, z):=\left(\begin{array}{c}
X_{s, t}(x) \\
\bar{Z}_{s, t}(x) z
\end{array}\right)
$$

Following the proof of Theorem 3.3 , we check that

$$
d F_{s, t}(\bar{x})=f(x) d\left(F \circ \bar{\chi}_{s, t}\right)(\bar{x})=-\left(\mathcal{G}_{s, t}(\bar{x}) d s+\mathcal{H}_{s, t}(\bar{x}) d \mathcal{U}_{s}\right)
$$

with the drift function

$$
\mathcal{G}_{s, t}(\bar{x}):=f(x) z\left(\nabla \overline{\mathbb{Q}}_{s, t}(g)(x)^{\prime} a_{s}(x)+\frac{1}{2} \operatorname{Tr}\left(\nabla^{2} \overline{\mathbb{Q}}_{s, t}(g)(x)^{\prime} \alpha_{s}(x)\right)\right)
$$

and the diffusion term

$$
\mathcal{H}_{s, t}(\bar{x}) d \mathcal{U}_{s}:=f(x) z\left(\nabla \overline{\mathbb{Q}}_{s, t}(g)(x)^{\prime} \sigma_{s}(x) d W_{s}+\overline{\mathbb{Q}}_{s, t}(g)(x) b_{s}(x)^{\prime} \beta_{s}^{-1} d Y_{s}\right)
$$

Applying the backward Itô-Ventzell formula [13] we check that

$$
d F_{s, t}\left(\bar{\chi}_{s}\right)=\left(d F_{s, t}\right)\left(\bar{\chi}_{s}\right)+\nabla F_{s, t}\left(\bar{\chi}_{s}\right)^{\prime} d \chi_{s}+\frac{1}{2} \operatorname{Tr}\left(\nabla^{2} F_{s, t}\left(\chi_{s}\right)^{\prime} \mathcal{A}_{t}\left(\bar{\chi}_{s}\right)\right) d s
$$

from which we conclude that

$$
\begin{aligned}
& d F_{s, t}\left(\bar{\chi}_{s}\right)=\bar{Z}_{s}\left(\nabla\left(\overline{\mathbb{Q}}_{s, t}(g)(x) f(x)\right)_{\mid x=X_{s}}^{\prime}-f\left(X_{s}\right) \bar{Z}_{s} \nabla \overline{\mathbb{Q}}_{s, t}(g)\left(X_{s}\right)^{\prime}\right) \sigma_{s}\left(X_{s}\right) d W_{s} \\
& -f\left(X_{s}\right) \bar{Z}_{s}\left(\nabla \overline{\mathbb{Q}}_{s, t}(g)\left(X_{s}\right)^{\prime} a_{s}\left(X_{s}\right) d s+\frac{1}{2} \operatorname{Tr}\left(\nabla^{2} \overline{\mathbb{Q}}_{s, t}(g)\left(X_{s}\right) \alpha_{s}\left(X_{s}\right)\right)\right) d s \\
& +\bar{Z}_{s}\left(\nabla\left(\overline{\mathbb{Q}}_{s, t}(g)(x) f(x)\right)_{\mid x=X_{s}}^{\prime} a_{s}\left(X_{s}\right) d s+\frac{1}{2} \operatorname{Tr}\left(\nabla^{2}\left(\overline{\mathbb{Q}}_{s, t}(g)(x) f(x)\right)_{\mid x=X_{s}}^{\prime} \alpha_{s}\left(X_{s}\right)\right)\right) d s
\end{aligned}
$$

We end the proof of (4.5) by simple integration.

To take the final step, we recall the integration by parts formula

$$
L_{t}(f g)=f L_{t}(g)+g L_{t}(f)+\Gamma_{L_{t}}(f, g)
$$


with the carré-du-champ (a.k.a. square field) operator $\Gamma_{L_{t}}$ associated with the generator $L_{t}$ defined by

$$
\Gamma_{L_{t}}(f, g):=(\nabla f)^{\prime} \alpha_{t} \nabla g
$$

Combining (4.5) with the above formula we check that

$$
\partial_{s}\left(\pi_{s}\left(f \bar{Q}_{s, t}(g)\right)\right)=\pi_{s}\left(L_{s}(f) \bar{Q}_{s, t}(g)\right)+\pi_{s}\left(\Gamma_{L_{s}}\left(\bar{Q}_{s, t}(g), f\right)\right)
$$

On the other hand, by an integration by parts we have

$$
\pi_{s}\left(\Gamma_{L_{s}}\left(\bar{Q}_{s, t}(g), f\right)\right)=-\sum_{i, j} \int p_{s}(x) \bar{Q}_{s, t}(g)(x) \frac{1}{p_{s}(x)} \partial_{x_{i}}\left(p_{s}(x) \alpha_{t}^{i, j} \partial_{x_{j}} f(x)\right) d x
$$

This ends the proof of the lemma.

Another approach for finding (4.5) is to use for any $u \in[s, t]_{h}$ the decomposition

$$
\begin{aligned}
\pi_{u+h} & \left(f \bar{Q}_{u+h, t}(g)\right)-\pi_{u}\left(f \bar{Q}_{u, t}(g)\right) \\
& =\pi_{u}\left(f\left(\bar{Q}_{u+h, t}-\bar{Q}_{u, t}\right)(g)\right)+\left(\pi_{u+h}-\pi_{u}\right)\left(f \bar{Q}_{u+h, t}(g)\right)
\end{aligned}
$$

Note that $\pi_{u}$ depends on the observations $\left(Y_{s}-Y_{0}\right)$ from $s=0$ up to time $s=u$, while the increment $\bar{Q}_{u, t}$ is computed backward in time and only depends on the observations $\left(Y_{s}-Y_{u}\right)$ from $s>u$ up to $s=t$. Conversely, $\pi_{u+h}$ depends on the observations $\left(Y_{s}-Y_{0}\right)$ from $s=0$ up to time $s=u+h$, while $\bar{Q}_{u+h, t}$ is computed backward in time and only depends on the observations $\left(Y_{s}-Y_{u+h}\right)$ from $s>u+h$, up to time $s=t$.

Following the two-sided stochastic integration calculus developped by Pardoux and Protter in [36] (see also [13] for extended versions to interpolating stochastic flows), combining the forward (3.8) with the backward equation (3.14), when $h \simeq 0$ we can check the approximation,

$$
\sum_{u \in[s, t]_{h}}\left\{\pi_{u+h}\left(f \bar{Q}_{u+h, t}(g)\right)-\pi_{u}\left(f \bar{Q}_{u, t}(g)\right)-\pi_{u}\left(L_{u}\left(f \bar{Q}_{u+h, t}(g)\right)-f L_{u}\left(\bar{Q}_{u+h, t}(g)\right)\right) h\right\} \simeq 0
$$

\subsection{Proof of Theorem 2.1}

With the definition in (4.1) we have,

$$
\pi_{t, s}(d x)=\left(\pi_{t} \mathcal{K}_{t, s}\right)(d x)=\pi_{s}(d x) \bar{Q}_{s, t}(1)(x)
$$

The formulation of the conditional distribution $\pi_{t, s}$ of $X_{s}$ given $\mathcal{Y}_{t}$ in (4.7) is rather well known, see e.g. Theorem 3.7 and Corollary 3.8 in [34], as well as equation (3.9) in [3]. The proof of this formula is a direct consequence of (4.1). With (4.2) we have,

$$
\pi_{t} \mathcal{K}_{t, s}=\pi_{t, u} \mathcal{K}_{u, s}=\pi_{t, s}
$$

Thus with $\mathcal{K}_{t, s}$ as defined in (4.1) we immediately have the transport equation in (2.1).

It remains to show that this integral operator (as defined in (4.1)) is also the Markov transition kernel of the backward diffusion flow in (2.2). The rest of the proof of Theorem 2.1] is a consequence of the duality formula (4.3) and Lemma 4.1.

Rewritten in a slightly different form, the duality formula (4.3) reads as follows,

$$
\mathbb{E}\left(f\left(X_{s}\right) g\left(X_{t}\right) \mid \mathcal{Y}_{t}\right)=\mathbb{E}\left(\mathcal{K}_{t, s}(f)\left(X_{t}\right) g\left(X_{t}\right) \mid \mathcal{Y}_{t}\right)
$$


This implies that

$$
\mathcal{K}_{t, s}(f)\left(X_{t}\right)=\mathbb{E}\left(f\left(X_{s}\right) \mid X_{t}, \mathcal{Y}_{t}\right)
$$

Finally, combining (4.4) with the duality formula (4.3) we have

$$
\pi_{t}\left(g \partial_{s} \mathcal{K}_{t, s}(f)\right)=-\pi_{t}\left(g \mathcal{K}_{t, s}\left(\mathcal{L}_{s, \pi_{s}}(f)\right)\right)
$$

Since the above formula is valid for any test function $g$ and $\pi_{t}$ has a bounded positive density, we check the backward Kolmogorov equation

$$
\partial_{s} \mathcal{K}_{t, s}(f)(x)=-\mathcal{K}_{t, s}\left(\mathcal{L}_{s, \pi_{s}}(f)\right)(x)
$$

with terminal condition $\mathcal{K}_{t, t}(f)=f$, when $s=t$, for almost every $x \in \mathbb{R}^{m}$ (and almost surely w.r.t. the law of the observation process from the origin up to the time $t$ ). Since both terms in (4.8) are continuous, the above equality holds for any $x \in \mathbb{R}^{m}$, almost surely.

We now complete the proof by showing that the integral operator $\mathcal{K}_{t, s}(x, d z)$ (defined in (4.1)) does indeed coincide with the transition kernel associated with the flow $\mathcal{X}_{t, s}(x)$ in (2.2). Firstly, observe that (4.8) coincides with the backward Kolmogorov equation (2.11) associated with the transition semigroup of the stochastic flow $\mathcal{X}_{t, s}(x)$. Denote this transition semigroup by $\overline{\mathcal{K}}_{t, s}(x, d z)$ temporarily.

By the semigroup properties of $\overline{\mathcal{K}}_{t, s}$, for any $s \leq u \leq t$ and any smooth function $f$ we have

$$
\partial_{u} \overline{\mathcal{K}}_{t, s}(f)=0=\partial_{u}\left(\overline{\mathcal{K}}_{t, u}\left(\overline{\mathcal{K}}_{u, s}(f)\right)\right)=-\overline{\mathcal{K}}_{t, u}\left(\mathcal{L}_{u, \pi_{u}}\left(\overline{\mathcal{K}}_{u, s} f\right)\right)+\overline{\mathcal{K}}_{t, u}\left(\partial_{u} \overline{\mathcal{K}}_{u, s}(f)\right)
$$

Choosing $u=t$ we obtain the forward equation

$$
\partial_{t} \overline{\mathcal{K}}_{t, s}(f)=\mathcal{L}_{t, \pi_{t}}\left(\overline{\mathcal{K}}_{t, s}(f)\right)
$$

Arguing as above, this implies that

$$
\partial_{u}\left(\mathcal{K}_{t, u}\left(\overline{\mathcal{K}}_{u, s}(f)\right)\right)=-\mathcal{K}_{t, u}\left(\mathcal{L}_{u, \pi_{u}}\left(\overline{\mathcal{K}}_{u, s} f\right)\right)+\mathcal{K}_{t, u}\left(\mathcal{L}_{u, \pi_{u}}\left(\overline{\mathcal{K}}_{u, s}(f)\right)\right)=0
$$

Integrating over the interval $[s, t]$ we check that $\mathcal{K}_{t, s}=\overline{\mathcal{K}}_{t, s}$. This ends the proof of Theorem 2.1 .

\section{References}

[1] B.D.O. Anderson. Fixed interval smoothing for nonlinear continuous time systems. Information and Control. vol. 20. pp. 294-300 (1972).

[2] B.D.O. Anderson. Reverse-time diffusion equation models. Stochastic Processes and their Applications. vol.12, no. 3. pp. 313-326 (1982).

[3] B.D.O. Anderson and I.B. Rhodes. Smoothing Algorithms for Nonlinear Finite-Dimensional Systems. Stochastics. vol. 9, no. 1-2. pp. 139-165 (1983).

[4] M. Arnaudon and P. Del Moral. A variational approach to nonlinear and interacting diffusions. Stochastic Analysis and Applications. vol. 37, no. 5. pp. 717-748 (2019).

[5] A. Bain and D. Crisan. Fundamentals of Stochastic Filtering. Springer (2009).

[6] A.E. Bryson and M. Frazier. Smoothing for linear and nonlinear dynamic systems. In Proceedings of the Optimum System Synthesis Conference. Technical Document Report No.: ASDTDR-63-119, Aeronautical Systems Division, Wright-Patterson Air Force Base, Ohio. pp. 353364 (1963). 
[7] P. Cattiaux and L. Mesnager. Hypoelliptic non-homogeneous diffusions. Probability Theory and Related Fields. vol. 123, no. 4, pp. 453-483 (2002).

[8] W.L. Chow. Über Systeme von linearen partiellen Differentialgleichungen erster Ordnung, Mathematische Annalen. vol. 117. pp. 98-105 (1939).

[9] T. Cass, M. Clark, and D. Crisan. The Filtering Equations Revisited. In Proc. of the Stochastic Analysis and Applications. pp. 129-162 (2014).

[10] G. Da Prato, J.L. Menaldi, and L. Tubaro. Some results of backward Ito formula. Stochastic Analysis and Applications. vol. 25, no. 3. pp. 679-703 (2007).

[11] G. Da Prato. Some remarks about backward Itô formula and applications. Stochastic Analysis and Applications. vol. 16, no. 6. pp. 993-1003 (1998).

[12] P. Del Moral and S. Penev. Stochastic Processes: From Applications to Theory. CRC Press (2017).

[13] P. Del Moral and S.S. Singh. A forward-backward stochastic analysis of diffusion flows. arXiv e-print, arXiv:1906.09145 (2019).

[14] H.G. Haussmann and E. Pardoux. Time reversal of diffusions. The Annals of Probability. vol. 14, no. 4. pp. 1188-205 (1986).

[15] L. Hörmander. Hypoelliptic second order differential equations. Acta Mathematica. vol. 119. pp. 147-171 (1967).

[16] T. Kailath, P. Frost. An innovations approach to least-squares estimation - Part II: Linear smoothing in additive white noise. IEEE Transactions on Automatic Control. vol. 13, no. 6. pp. 655-660 (1968).

[17] G. Kallianpur and C. Striebel. Estimation of stochastic systems: arbitrary system process with additive noise observation errors. The Annals of Mathematical Statistics. vol. 39, no. 3. pp. 785-801 (1968).

[18] G. Kallianpur and C. Striebel. Stochastic differential equations occurring in the estimation of continuous parameter stochastic processes. Theory of Probability \& Its Applications. vol. 14, no. 4. pp. 567-594 (1969).

[19] N.V. Krylov and B.L. Rozowskii. On the Cauchy problem for linear stochastic partial differential equations. Mathematics of the USSR-Izvestiya. vol. 11, no. 4. (1977).

[20] N.V. Krylov and B.L. Rozowskii. Conditional distributions of diffusion processes. Mathematics of the USSR-Izvestiya. vol. 12, no. 2. (1978).

[21] N.V. Krylov and B.L. Rozowskii. On the first integrals and Liouville equations for diffusion processes. In Stochastic Differential Systems: Proc. 3rd SFSP-WG 7/1. Part of the Lecture Notes in Control and Information Sciences. vol. 36. pp. 117-125. Springer (1981).

[22] H. Kunita. Asymptotic behavior of the nonlinear filtering errors of Markov processes. Journal of Multivariate Analysis. vol. 1, no. 4. pp 365-393 (1971). 
[23] H. Kunita. Stochastic partial differential equations connected with non-linear filtering. In Nonlinear Filtering and Stochastic Control. Part of the Lecture Notes in Mathematics. vol. 972. pp. 100-169. Springer (1982).

[24] H. Kunita. On backward stochastic differential equations. Stochastics. vol. 6, no. 3-4. pp. 293313 (1982).

[25] H. Kunita. First order stochastic partial differential equations. In Stochastic Analysis: Proc. of the Taniguchi International Symposium on Stochastic Analysis. pp. 249-269 (1984).

[26] H.J. Kushner. On the differential equations satisfied by conditional probability densities of Markov processes, with applications. Journal of the Society for Industrial and Applied Mathematics, Series A: Control. vol. 2, no. 1. pp. 106-119 (1964).

[27] C.T. Leondes, J.B. Peller, and E.B. Stear. Nonlinear smoothing theory. IEEE Transactions on Systems Science and Cybernetics. vol. 6, no. 1. pp. 63-71 (1970).

[28] J.S. Meditch. A Survey of Data Smoothing for Linear and Nonlinear Dynamic Systems. Automatica. vol. 9. pp. 151-162 (1973).

[29] D. Michel. Régularité des lois conditionnelles en théorie du filtrage non-linéaire et calcul des variations stochastique. Journal of Functional Analysis. vol. 41, no. 1. pp. 8-36 (1981).

[30] A. Millet, D. Nualart, and M. Sanz. Integration by parts and time reversal for diffusion processes. The Annals of Probability. vol. 17, no.1. pp. 208-238 (1989).

[31] E. Pardoux. Equations aux dérivées partielles stochastiques non linéaires monotones. Doctoral Thesis, Université Paris XI, Orsay (1975).

[32] E. Pardoux. Stochastic partial differential equations and filtering of diffusion processes. Stochastics. vol. 3, no. 1-4. pp. 127-167 (1980).

[33] E. Pardoux, Non-linear filtering, prediction and smoothing, in M. Hazewinkel and J. C. Willems (eds.), Stochastic Systems: The Mathematics of Filtering and Identijication and Applications, Reidel, Dordrecht (1981).

[34] E. Pardoux. Équations du filtrage non linéaire de la prédiction et du lissage. Stochastics. vol. 6, no. 3-4. pp. 193-231 (1982).

[35] E. Pardoux and S. Peng. Adapted solution of a backward stochastic differential equation. Systems \& Control Letters. vol. 14, no. 1. pp. 55-61 (1990).

[36] E. Pardoux and P. Protter. A two-sided stochastic integral and its calculus. Probability Theory and Related Fields. vol. 76, no. 1. pp. 15-49 (1987).

[37] H.E. Rauch, F. Tung, and C.T. Striebel. Maximum likelihood estimates of linear dynamic systems. AIAA Journal. vol. 3, no. 8. pp. 1445-1450 (1965).

[38] P.K. Rashevskii. About connecting two points of complete non-holonomic space by admissible curve (in Russian). Uch. Zapiski Ped. Instit. Libknexta. vol. 2. pp 83-94 (1938).

[39] M. Rutkowski. A simple proof for the Kalman-Bucy smoothed estimate formula. Statistics \& Probability Letters. vol. 17, no. 5. pp. 377-385 (1993). 
[40] J. M. Steele. Stochastic Calculus and Financial Applications. Springer (2012).

[41] A.Y. Veretennikov. On backward filtering equations for SDE systems (direct approach). In Stochastic Partial Differential Equations: Part of the Lecture Notes Series. vol. 216. pp. 304311. London Mathematical Society (1995).

[42] T. Yang, P.G. Mehta, and S.P. Meyn. Feedback particle filter. IEEE Transactions on Automatic Control. vol. 58, no. 10. pp. 2465-2480 (2013).

[43] T. Yang, R.S. Laugesen, P.G. Mehta, and S.P. Meyn. Multivariable feedback particle filter. Automatica. vol. 71. pp. 10-23 (2016).

[44] M. Zakai. On the Optimal Filtering of Diffusion Processes. Zeitschrift für Wahrscheinlichkeitstheorie und Verwandte Gebiete. vol. 11, no. 3. pp. 230-243 (1969). 